\title{
Corneal incision architecture: VICTUS femtosecond laser vs manual keratome
}

Renan Rodrigues'

Myrna Serapião Dos

Santos ${ }^{1,2}$

Rachel E Silver ${ }^{3}$

Mauro Campos ${ }^{1,2}$

Rachel LR Gomes 1,2

'Department of Ophthalmology, Federal University of Sao Paulo, Sao Paulo, Brazil; ${ }^{2}$ Cataract Department, Hospital de Olhos Paulista, Sao Paulo, Brazil; ${ }^{3}$ Friedman School of Nutrition Science and Policy, Tufts University, Boston, MA, USA
This article was published in the following Dove Medical Press journal: Clinical Ophthalmology

Purpose: To evaluate anatomical differences between laser (VICTUS) and manual constructed phacoemulsification incisions.

Setting: Study was conducted at the Hospital de Olhos Paulista, Sao Paulo, Brazil.

Design: This is a prospective, observational, consecutive nonrandomized cohort.

Patients and methods: Sixteen patients (18 eyes) had phacoemulsification surgery, nine eyes had manual incisions and nine eyes had femtosecond incisions. All incisions were performed by the same surgeon. All incisions were evaluated at the 1st and 30th postoperative day with anterior segment optical coherence tomography to evaluate anatomical features. Inclusion criterion was presence of low visual acuity due to cataract. Exclusion criteria were other ocular pathologies, previous trauma, or corneal wound healing disease, as any intra or postoperative complication. Three different corneal specialists analyzed wound architecture features (epithelial or endothelial gap, incision edema, number of planes, Descemet's detachment, and loss of wound sealing) at both visits. ImageJ (National Institutes of Health) software was also used to analyze anatomical features of incisions. Results: Almost all incisions had edema at the first postoperative day. All femto incisions were triplanar, as intended, unlike manual incisions, which showed two uniplanar incisions $(P=0.009)$. All laser incisions presented partial loss of wound sealing, while five keratome incisions had no loss of wound sealing at the first postoperative day $(P=0.03)$.

Conclusion: These findings support the hypothesis that laser constructed incisions are more precisely constructed than manual, although corneal wound healing was similar between the two groups at the 30th postoperative day, with a tendency to a faster anatomical improvement in the keratome group.

Keywords: phacoemulsification, cataract, femtosecond laser, optical coherence tomography, cornea

\section{Introduction}

Cataract is the leading cause of blindness worldwide. According to global estimates in 2010, 10.8 million people were blind and 35.1 million experienced visual impairment as a result of cataract in at least one eye, translating to $33.4 \%$ of all blindness and $18.4 \%$ of people suffering from visual impairment. ${ }^{1}$ This increasing prevalence of cataract indicates a significant public health issue, despite the wide availability of extraction methods that reverse the impact of cataract. The most popular extraction procedure is phacoemulsification. This method is characterized by the creation of a small corneal incision using a keratome, followed by emulsification of the natural lens and intraocular lens (IOL) implantation.

Recent technology allows for the use of femtosecond lasers to perform the incision as well as the capsulorhexis and nucleus fragmentation. Femtosecond laser-assisted cataract surgery (FLACS) has been demonstrated to improve the accuracy of each of
Correspondence: Renan Rodrigues Department of Ophthalmology, Federal University of Sao Paulo, Paula Alves Street, 25, apt 302, Natal/RN, 59.022 135, Brazil

Tel +558440088300

Email renanrodrigues.med@gmail.com (c)
hereby accept the Terms. Non-commercial uses of the work are permitted without any further permission from Dove Medical Press Limited, provided the work is properly attributed. For permission for commercial use of this work, please see paragraphs 4.2 and 5 of our Terms (https://www.dovepress.com/terms.php). 
these steps. Several studies have demonstrated the safety and effectiveness of FLACS. ${ }^{2-4}$ Specific indications of FLACS remains unclear, although it appears that it creates more predictable and replicable incisions, as well as superior wound closure. ${ }^{3}$ This method can therefore be related to the incidence of anterior capsule tears, posterior capsule tears, and other complications, and they are more frequent at the beginning of the learning curve. ${ }^{2}$

Given that the benefits of extraction methods such as FLACS include improved surgical outcomes, it is clear that precision in the architecture of the corneal incisions is of utmost importance. An incision with the correct size and length can facilitate the success of the subsequent surgical steps. The incision enables flow through the cannula sleeve, which is necessary for an effective emulsification and adequate incision temperature. Moreover, a well-constructed incision allows for better wound sealing at the end of the procedure, avoiding the influx of particles and microorganisms that can cause endophthalmitis.

Anterior segment optical coherence tomography can be used to evaluate aspects of wound architecture following the extraction in vivo. Anterior segment optical coherence tomography provides significant information related to the success of the surgical process, including the incision angle, length, corneal thickness, epithelial gap, endothelial gap, and Descemet's detachment. $^{5}$

In this study, we aimed to evaluate morphologic features associated with wound architecture following cataract extraction using laser and keratome incisions. We assessed the difference of the features at 1 and 30 days postprocedure in order to determine anatomical changes after phacoemulsification. We hypothesized that cataract extraction performed using FLACS would be more precise and that these patients would have better improvements in recovery.

\section{Patients and methods}

This prospective observational cohort study was conducted at the Hospital de Olhos Paulista (Sao Paulo, Brazil). All procedures were performed between May and July 2015, and all extractions were performed by the same surgeon (RLRG). Written informed consent was obtained from all participants, and the study was reviewed by an ethics committee.

All patients received a complete ocular examination in order to confirm the presence of cataract. Exclusions prior to surgery were made based on the following criteria: the detection of a concomitant ocular pathology, history of previous ocular trauma, history of surgical or laser treatment, history of concomitant ocular or medical conditions that could affect corneal wound healing. Additional exclusions were made following the extraction procedures: those requiring enlargement of the corneal incision during surgery, those with intraoperative or postoperative complications that could potentially affect the cornea, and those requiring placement of a suture at the end of surgery. The final sample size included 16 patients (18 eyes). Nine eyes underwent FLACS using a VICTUS laser (Bausch \& Lomb Incorporated, Bridgewater, NJ, USA) and nine eyes had a manual blade incision using a 2.75 keratome (Mani, Utsunomiya, Japan). Triplanar manual incisions were intended for all eyes receiving the manual incision. In the FLACS group, a $2.75 \mathrm{~mm} 3$-plane incision was created with a VICTUS femtosecond laser. The laser parameters are reviewed in Table 1.

After phacoemulsification and cortical aspiration in both groups, the IOL was implanted in the capsular bag without corneal wound enlargement. The corneal wounds were not hydrated, and Seidel was tested using a swab at the end of the procedure. Postoperative prescription was the same for all surgeries.

Table I Parameters associated with the VICTUS $2.7 \mathrm{sw}$ femtosecond laser

\begin{tabular}{l|l|l|l}
\hline Capsulotomy & Adjustment & Corneal incisions & Primary/secondary \\
\hline Diameter & 4800 & Tunnel width & $2800 / I 100$ \\
Height & 800 & Axis (primary) & $100 / 25$ \\
Spacing & $6.0 / 4.0$ & Cut overlap & 10 \\
Energy & 7000 & Spot/line spacing & $5.5 / 2.0$ \\
Lens fragmentation & & Energy & 2000 \\
$\quad$ Spot/line spacing & $10.0 / I 0.0$ & Anterior/posterior bonus & $150 /-150$ \\
Radial cuts & 6 & Primary incision & Plane I/2/3 \\
Radial outer diameter & 7000 & Depth & $-200 /-400 /-900$ \\
Phaco & Stellaris & Side cut angle & $70 / 1000 / 70$ \\
Technique & Preslice & Secondary incision & Plane I/2/3 \\
Energy & 8400 & Depth & $-100 /-9 / 0 /-1000$ \\
& & Side cut angle & $40 / 1500 / 70$ \\
\hline
\end{tabular}




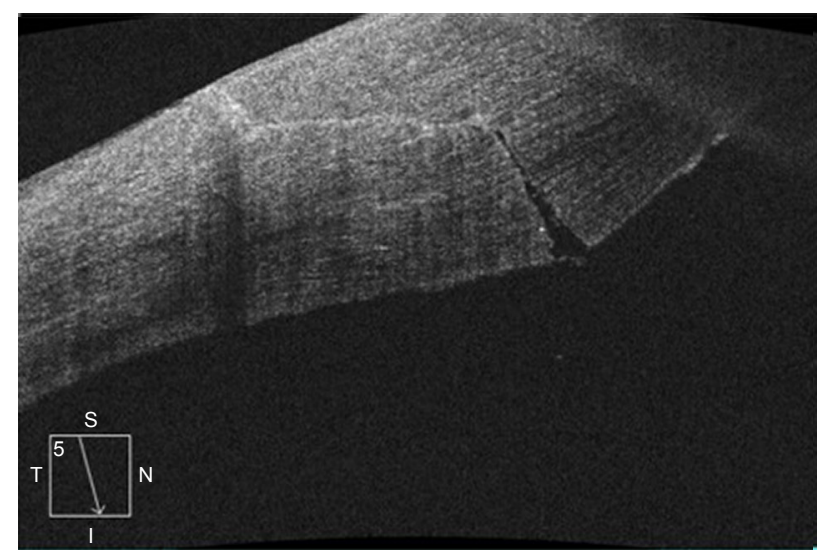

Figure I Femtosecond created incision.

Note: Note that even with a triplanar construction, loss of wound sealing $<50 \%$ is present at the first postoperative day.

Wound architecture was evaluated using anterior segment Cirrus OCT (Carl Zeiss Meditec Inc, Dublin, CA, USA). Images were captured at 1 st and 30 th postoperative day. Three masked ophthalmologists evaluated the images to qualitatively analyze the morphologic wound architecture features. These features included epithelial gap (present or absent), endothelial gap (present or absent), incision edema (present or absent), number of planes (uniplanar, biplanar, or triplanar), Descemet's detachment (present or absent), and loss of wound sealing (total if $>50 \%$, partial if $<50 \%$, or absent). Features were assessed at both time points. A quantitative analysis of the images was performed using ImageJ software as demonstrated in Figure 1 and Table 2.

Table 2 Morphologic characteristics of incisions as assessed by ImageJ software

\begin{tabular}{|c|c|c|c|}
\hline Cornea & Length $(\mu)$ & Gap size $(\mu)$ & Thickness $(\mu)$ \\
\hline 01 & 352.0 & 26.6 & 270.0 \\
\hline 02 & 344.0 & 34.7 & 246.0 \\
\hline 03 & 234.7 & 34.0 & 280.1 \\
\hline 04 & 402.7 & 25.0 & 220.1 \\
\hline 05 & 448.0 & 46.0 & 229.0 \\
\hline 06 & 284.0 & 0.0 & 191.6 \\
\hline 07 & 432.0 & 63.5 & 237.5 \\
\hline 08 & 326.7 & 24.0 & 210.7 \\
\hline 09 & 309.3 & 31.5 & 202.1 \\
\hline 10 & 266.7 & 36.1 & 269.5 \\
\hline II & 494.7 & 109.8 & 275.0 \\
\hline 12 & 288.0 & 0.0 & 200.0 \\
\hline 13 & 242.7 & 23.9 & 217.3 \\
\hline 14 & 172.0 & 23.3 & 168.1 \\
\hline 15 & 338.7 & 30.7 & 253.9 \\
\hline 16 & 340.0 & 14.7 & 213.5 \\
\hline 17 & 388.0 & 22.7 & 273.7 \\
\hline 18 & 305.3 & 47.2 & 225.0 \\
\hline
\end{tabular}

Quantitative features evaluated included corneal thickness, gap size, and incision length.

The presence of each wound architecture feature was qualitatively assessed at 1 and 30 days postprocedure. In order to compare the presence of these features for the two surgical groups, the observations made by the three ophthalmologists were combined using the assessment with the highest frequency. Each feature was assigned to a specific strata based on the concordant assessment of at least two of the readers. The agreement between the three ophthalmologists was evaluated for the qualitative assessments at each of the two time points.

Comparisons between the VICTUS and keratome surgical groups at each time point were made using the Fisher's exact test (Table 3). The quantitative wound architecture features were measured at 1 day postprocedure for both surgical methods. The mean measurements for the VICTUS and keratome groups were compared using Student's $t$-test for independent samples, with unequal variances assumed (Table 4). A $P$-value of 0.05 was determined a priori as the threshold for statistical significance. All statistical analyses were conducted using SAS 9.4 (SAS Institute Inc., Cary, NC, USA).

The study adhered to the tenets of the Declaration of Helsinki and was approved by the ethics committee (Ethics Committee of the Hospital Sao Paulo - Federal University of Sao Paulo). All patients provided written informed consent regarding their participation in the study.

\section{Results}

A total of 18 eyes from 16 patients were included in the qualitative and quantitative assessment of morphologic features of wound architecture following cataract extraction by two distinct surgical techniques. Assessment of these features was conducted at each visit by three ophthalmologists, with an overall rate of agreement of $88 \%$. The distribution of these features at 1 and 30 days postoperatively in the FLACS and keratome groups is presented in Table 3.

At day 1, differences were observed in the assessment of the number of incision planes $(P=0.009)$. The VICTUS group was determined to have primarily triplanar incisions $(100 \%)$, while the keratome displayed more variation in the assessment of the incision planes. Similar differences were observed at 30 days postprocedure $(P<0.001)$. Significant differences were also observed in the evaluation of the loss of incision wound sealing at day $1(P=0.03)$. The VICTUS group was assessed to have partial loss of wound sealing (100\%), whereas the keratome group was determined to have either no loss or partial loss (55\% or $44 \%$, respectively). 
Table 3 Distribution of wound architecture features among VICTUS and keratome surgical groups at I and 30 days postprocedure

\begin{tabular}{|c|c|c|c|c|c|c|}
\hline \multirow[t]{3}{*}{ Incision parameter } & \multicolumn{3}{|c|}{ One day postprocedure } & \multicolumn{3}{|c|}{ Thirty days postprocedure } \\
\hline & \multirow{2}{*}{$\begin{array}{l}\text { VICTUS n (\%) } \\
n=9\end{array}$} & \multirow{2}{*}{$\begin{array}{l}\text { Keratome } \mathrm{n}(\%) \\
\mathrm{n}=9\end{array}$} & \multirow[t]{2}{*}{$P$-value ${ }^{b}$} & \multirow{2}{*}{$\begin{array}{l}\text { VICTUS n (\%) } \\
n=9\end{array}$} & \multirow{2}{*}{$\begin{array}{l}\text { Keratome n (\%) } \\
n=9\end{array}$} & \multirow[t]{2}{*}{$P$-value ${ }^{b}$} \\
\hline & & & & & & \\
\hline \multicolumn{7}{|l|}{ Edema } \\
\hline No & I (II.I) & $2(22.2)$ & 0.99 & $9(100.0)$ & $9(100.0)$ & - \\
\hline Yes & 8 (88.9) & 7 (77.8) & & $0(0.0)$ & $0(0.0)$ & \\
\hline \multicolumn{7}{|l|}{ Incision } \\
\hline Uniplanar & $0(0.0)$ & $2(22.2)$ & 0.009 & $0(0.0)$ & $2(22.2)$ & $<0.001$ \\
\hline Biplanar & $0(0.0)$ & $4(44.4)$ & & $0(0.0)$ & $5(55.6)$ & \\
\hline Triplanar & $9(100.0)$ & $3(33.3)$ & & $9(100.0)$ & I (II.I) & \\
\hline \multicolumn{7}{|l|}{ Epithelial gap } \\
\hline No & $8(88.9)$ & 7 (77.8) & 0.99 & $9(100.0)$ & $9(100.0)$ & - \\
\hline Yes & I (II.I) & $2(22.2)$ & & $0(0.0)$ & $0(0.0)$ & \\
\hline \multicolumn{7}{|l|}{ Endothelial gap } \\
\hline No & $0(0.0)$ & $2(22.2)$ & 0.47 & $4(44.4)$ & 7 (77.8) & 0.33 \\
\hline Yes & $9(100.0)$ & 7 (77.8) & & $5(55.6)$ & $2(22.2)$ & \\
\hline \multicolumn{7}{|l|}{ Descemet's detachment } \\
\hline No & $6(66.7)$ & $3(33.3)$ & 0.35 & $9(100.0)$ & 8 (88.9) & 0.99 \\
\hline Yes & $3(33.3)$ & $6(66.7)$ & & $0(0.0)$ & I (II.I) & \\
\hline \multicolumn{7}{|c|}{ Loss of incision wound sealing } \\
\hline No loss & $0(0.0)$ & $5(55.6)$ & 0.03 & $7(77.8)$ & $7(77.8)$ & 0.99 \\
\hline Partial loss (<50\%) & $9(100.0)$ & $4(44.4)$ & & $2(22.2)$ & $2(22.2)$ & \\
\hline Total loss $(>50 \%)$ & $0(0.0)$ & $0(0.0)$ & & $0(0.0)$ & $0(0.0)$ & \\
\hline \multicolumn{7}{|l|}{ Incision type } \\
\hline Laser & $9(100.0)$ & $0(0.0)$ & $<0.001$ & $9(100.0)$ & $0(0.0)$ & $<0.001$ \\
\hline Blade & $0(0.0)$ & $9(100.0)$ & & $0(0.0)$ & $9(100.0)$ & \\
\hline
\end{tabular}

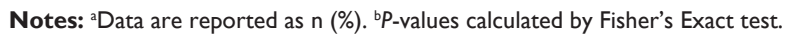

This result was not retained during evaluation at day 30 , and there was no significant difference in the assessment of wound sealing between the two groups. As expected, the incision type was accurately evaluated and was significantly different between the two surgical groups $(P<0.001)$. No significant differences between the groups at either time point were observed for the assessment of edema, epithelial gap, endothelial gap, or Descemet's detachment.

Quantitative features were also evaluated at 1 day postoperative for the VICTUS and keratome groups. Comparisons of mean measurements for incision thickness, gap size, and length did not significantly differ between the two groups, although the cornea tended to be thicker and length was smaller in the VICTUS group $(\mu=242 \mu \mathrm{m}$ and $310 \mu \mathrm{m}$, respectively).

Table 4 Distribution of quantitative wound architecture features among VICTUS and keratome surgical groups at I day postprocedure

\begin{tabular}{l|l|l|l}
\hline Incision parameter & $\begin{array}{l}\text { VICTUS, } \\
\text { mean } \pm \text { SD }^{\mathbf{a}}\end{array}$ & $\begin{array}{l}\text { Keratome, } \\
\text { mean } \pm \mathbf{S D}\end{array}$ & \multirow{2}{*}{-value ${ }^{\mathrm{b}}$} \\
\cline { 2 - 3 } & $\mathbf{n}=\mathbf{9}$ & $\mathbf{n}=\mathbf{9}$ & \\
\hline Corneal thickness & $242.7 \pm 27.7$ & $222.1 \pm 35.9$ & 0.19 \\
Gap size & $32.2 \pm 7.1$ & $33.8 \pm 35.0$ & 0.89 \\
Length & $310.7 \pm 55.3$ & $352.6 \pm 99.5$ & 0.29 \\
\hline
\end{tabular}

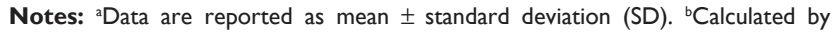
Student's $t$-test for independent samples, unequal variances assumed.

\section{Discussion}

Our study of wound architecture features following cataract extraction by two distinct methods revealed that laser incisions were more precisely constructed than manual incisions. All laser incisions were triplanar, as intended, while manual incisions showed significantly more variety $(P=0.001)$. While regarding qualitative analysis, we found that qualitative assessments by trained ophthalmologists demonstrated a high degree of agreement (88\%). However, a few unexpected results must be discussed.

Cornea wound sealing was examined at the postoperative day 1 in order to search for signs of leakage or incision instability, as this may be correlated with a higher probability of endophthalmitis. At postoperative day 30, incisions were evaluated regarding its final morphological aspect. At the previous postoperative days 7 and 14, there still may occur anatomical improvement of the incision, which can vary among patients, with limited clinical relevance.

In the VICTUS group, all incisions were assessed to an endothelial gap at the 1 st postoperative day, and at 30th postoperative day, $55 \%$ of incisions still presented endothelial gap. Grewal and Basti previously showed that, using Catalys Precision Laser System, OptiMedica Corp., there was a significant lower endothelial gap in the laser group than in the keratome group. ${ }^{6}$ Furthermore, it is important to note that among patients undergoing manual extraction, the endothelial 
gap resumed earlier, although this result was not statistically significant.

This observation is particularly relevant as uniplanar incisions can be unstable and less safe than triplanar incisions, resulting in a higher risk of loss of integrity and a less effective barrier against pathogens in the early postoperative period, as previous demonstrated by Fine et al. ${ }^{7}$

Descemet's detachment at the first postoperative day was more frequently observed in the keratome group, which is consistent with results observed previously. ${ }^{6}$ Laser incisions may therefore be beneficial as Descemet's membrane detachment is related to local or total corneal edema. These forms of edema can result in long-term complications, such as lower best-corrected visual acuity. Another study had previously demonstrated similar results between VICTUS and manual phacoemulsification, although incision structure was not analyzed, only central corneal thickness. ${ }^{8}$

At the first postoperative day, manual incisions had less loss of wound sealing than laser ones, as illustrated in Figure 2, whereas laser incisions were associated with partial wound sealing and this finding was statistically significant $(P=0.03)$. Although this difference was not observed at the 30 th postoperative day, this observation prompts a discussion about safety during the earlier postoperative period as the risk of endophthalmitis is higher. As a result, in this study, it would appear that manual incisions showed to be safer compared with those performed by this femtosecond laser, although this finding requires confirmation in a larger population. Given the difference in postoperative wound sealing, manual incision showed a faster anatomical improvement.

This study has a few limitations: relative small number of participants, variation of implanted IOL among patients, consequently with a variation on injector mechanism. Regarding the last, it was minimized by avoiding incision

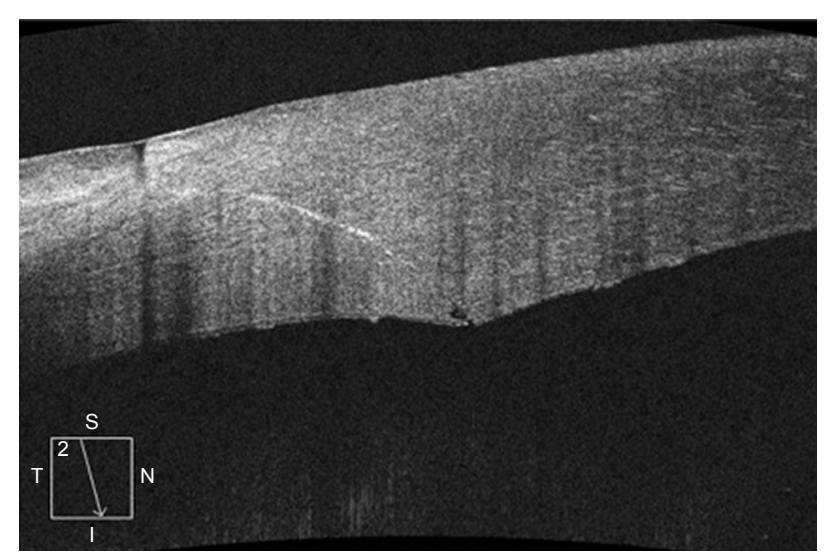

Figure 2 Keratome created incision.

Note: Fast anatomical improvement, with no loss of wound sealing, at the first postoperative day is noted. enlargement during implantation. Although the number of participants was small, results were consistent and allowed adequate analysis.

Differences in anatomical improvement after manual compared with VICTUS femtosecond laser constructed incisions require additional study. It is clear that the laser can be highly beneficial, but findings evidenced by this study revealed that we need to go further and analyze the anatomical results of each femtosecond laser platform available, as had been investigated by another studies. ${ }^{9,10}$ Therefore, although studies have demonstrated that FLACS is safe and can be a more precise way to perform cataract extraction, ${ }^{11}$ with less energy dispended and possibly less endothelial cells loss, the higher cost of this procedure remains a barrier to its widespread use.

\section{Disclosure}

The authors report no conflicts of interest in this work.

\section{References}

1. Khairallah M, Kahloun R, Bourne R, et al; Vision Loss Expert Group of the Global Burden of Disease Study. Number of People Blind or Visually Impaired by Cataract Worldwide and in World Regions, 1990 to 2010. Invest Opthalmol Vis Sci. 2015;56(11):6762-6769.

2. Roberts TV, Lawless M, Bali SJ, Hodge C, Sutton G. Surgical outcomes and safety of femtosecond laser cataract surgery: a prospective study of 1500 consecutive cases. Ophthalmology. 2013;120(2):227-233.

3. Masket S, Sarayba M, Ignacio T, Fram N. Femtosecond laser-assisted cataract incisions: architectural stability and reproducibility. J Cataract Refract Surg. 2010;36(6):1048-1049.

4. Nagy Z, Takacs A, Filkorn T, Sarayba M. Initial clinical evaluation of an intraocular femtosecond laser in cataract surgery. J Refract Surg. 2009;25(12):1053-1060.

5. Lee H, Kim EK, Kim HS, Kim TI. Fourier-domain optical coherence tomography evaluation of clear corneal incision structure according to blade material. J Cataract Refract Surg. 2014;40(10):1615-1624.

6. Grewal DS, Basti S. Comparison of morphologic features of clear corneal incisions created with a femtosecond laser or a keratome. J Cataract Refract Surg. 2014;40(4):521-530.

7. Fine IH, Hoffman RS, Packer M. Profile of clear corneal cataract incisions demonstrated by ocular coherence tomography. J Cataract Refract Surg. 2007;33(1):94-97.

8. Mursch-Edlmayr AS, Bolz M, Luft N, et al. Intraindividual comparison between femtosecond laser-assisted and conventional cataract surgery. J Cataract Refract Surg. 2017;43(2):215-222.

9. Grewal DS, Schultz T, Basti S, Dick HB. Femtosecond laser-assisted cataract surgery - current status and future directions. Surv Ophthalmol. 2016;61(2):103-131.

10. Bm W, Williams GP, Tan A, Mehta JS. A comparison of different operating systems for femtosecond lasers in cataract surgery. JOphthalmol. 2015;2015:616478.

11. Ang RET, Quinto MMS, Cruz EM, Rivera MCR, Martinez GHA. Comparison of clinical outcomes between femtosecond laser-assisted versus conventional phacoemulsification. Eye Vis (Lond). 2018;5:8. 


\section{Publish your work in this journal}

Clinical Ophthalmology is an international, peer-reviewed journal covering all subspecialties within ophthalmology. Key topics include: Optometry; Visual science; Pharmacology and drug therapy in eye diseases; Basic Sciences; Primary and Secondary eye care; Patient Safety and Quality of Care Improvements. This journal is indexed on

Submit your manuscript here: http://www.dovepress.com/clinical-ophthalmology-journal
PubMed Central and CAS, and is the official journal of The Society of Clinical Ophthalmology (SCO). The manuscript management system is completely online and includes a very quick and fair peer-review system, which is all easy to use. Visit http://www.dovepress.com/ testimonials.php to read real quotes from published authors. 